\title{
Estimation of Galactic Model Parameters In High Latitude With SDSS and SCUSS
}

\author{
Yunpeng Jia and Cuihua Du \\ School of Physics, University of Chinese Academy of Sciences \\ email: jiayunpeng11@mails.ucas.ac.cn
}

\begin{abstract}
Using star counts method, we estimated the Galactic structure parameters in high latitude field $\left(50^{\circ} \leqslant l \leqslant 55^{\circ},-46^{\circ} \leqslant b \leqslant-44^{\circ}\right), 10 \mathrm{deg}^{2}$ field with Sloan Digital Sky Survey (SDSS) and South Galactic Cap of U-band Sky Survey (SCUSS), to explore their possible variations with absolute magnitude. Here we just considered three components: double exponential thin disk and thick disk and a de Vaucouleurs halo. And these parameters were obtained by minimising $\chi^{2}$.
\end{abstract}

Keywords. Galaxies: structure, Galaxies: fundamental parameters

Our study show these parameters are depend on absolute magnitude (see Table 1). The thin disk and halo show more regular tend with absolute magnitude change than thick disk. The scale-height of thin disk $h_{1}$ increased and local density $n_{1}$ decreased, due to the two parameters depend on each other, when the sample stars become bright. This means faint stars in thin disk, like to stay close to the plane than the bright. And faint stars have lager local density than bright one, which hold for thin disk and halo. While thick disk shows complex trend, which may caused by the degeneracy and the dependence between some parameters. It is cleared that different type stars could have different specific distributions. A possible explanation to this is that absolute faint stars had a longer lifetime, so experienced longer dynamic process which made them closer to the plane, than that of bright one.

Table 1. Parameters for three components

\begin{tabular}{ccccccccc}
\hline $\begin{array}{c}\mathbf{M} \\
(\mathrm{mag})\end{array}$ & $\begin{array}{c}\boldsymbol{n}_{\mathbf{1}} \\
\left(\text { star } / k p c^{3}\right)\end{array}$ & $\begin{array}{c}\boldsymbol{h}_{\mathbf{1}} \\
(p c)\end{array}$ & $\begin{array}{c}\boldsymbol{n}_{\mathbf{2}} \\
\left(\text { star } / k p c^{3}\right)\end{array}$ & $\begin{array}{c}\boldsymbol{h}_{\mathbf{2}} \\
(p c)\end{array}$ & $\begin{array}{c}\boldsymbol{n}_{\mathbf{2}} / \boldsymbol{n}_{\mathbf{1}} \\
(\text { percent })\end{array}$ & $\begin{array}{c}\boldsymbol{n}_{\mathbf{3}} \\
\left(\text { star } / k p c^{3}\right)\end{array}$ & $\boldsymbol{\kappa}$ & $\begin{array}{c}\boldsymbol{n}_{\mathbf{3}} / \boldsymbol{n}_{\mathbf{1}} \\
(\text { percent })\end{array}$ \\
\hline $4-5$ & & & $3.70 e^{5}$ & 560.0 & & $1.39 e^{3}$ & 0.8 & \\
\hline $5-6$ & $1.00 e^{6}$ & 350.0 & $1.50 e^{5}$ & 765.0 & 15.0 & $2.45 e^{3}$ & 0.6 & 0.245 \\
\hline $6-8$ & $3.00 e^{6}$ & 220.0 & $5.25 e^{5}$ & 555.0 & 17.5 & $7.39 e^{3}$ & 0.5 & 0.246 \\
\hline $8-10$ & $1.09 e^{7}$ & 178.0 & & & & & & \\
\hline $10-13$ & $1.93 e^{7}$ & 102.0 & & & & & & \\
\hline $4-13$ & $1.46 e^{7}$ & 195.0 & $1.24 e^{6}$ & 582.5 & 8.5 & $5.84 e^{3}$ & 0.6 & 0.04 \\
\hline
\end{tabular}

Notes: Suffix 1,2,3, denote thin disk, thick disk, halo,respectively.

\section{References}

Bilir S., Karaali S., Gilmore G. 2006, MNRAS, 336,1295

Jurić M., Ǐvezić Z., et al. 2008, ApJ, 673, 864

Phleps S., Meisenheimer K., Fuchs B., \& Wolf C. 2000, A\&A, 356, 108 